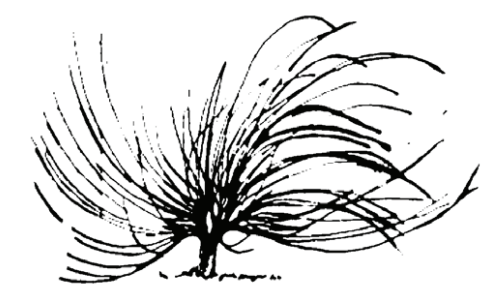

\title{
Automatización de la Educación: La Ejecución Pragmática del Proyecto Neoliberal en las Políticas Educativas Estatales
}

\author{
Emanuel Soto Cabezas ${ }^{1}$ \\ Universidad Nacional \\ Heredia, Costa Rica \\ emasoto@gmail.com
}

\begin{abstract}
Resumen
Este ensayo es una breve disertación sobre los nuevos intereses que los Estados nacionales han definido para sus políticas educativas. En una sociedad cada vez más orientada a disminuir el tamaño del Estado, la funcionalidad de la agenda educativa está definiendo cuales son los nuevos perfiles profesionales que se incentivan, así como, las habilidades sociales que se consideran oportunas al modelo económico vigente.
\end{abstract}

Palabras clave: políticas públicas, educación, mercado, neoliberalismo, telemática

\begin{abstract}
This essay is a short presentation of the new interests that nation states have defined for their educational policies. In a society increasingly oriented towards reducing the size of the state, the functionality of the educational agenda is defining which new professional profiles are being promoted as well as the social skills that are considered appropriate for the current economic model.
\end{abstract}

Recibido: 27 de febrero de 2014 - Aprobado: 18 de agosto de 2014

1 Docente y politólogo costarricense (1985). Máster en Estudios Latinoamericanos con énfasis en Cultura y Desarrollo de la Universidad Nacional de Costa Rica. 
Keywords: public policies, education, market, neoliberalism, telematics

\section{Introducción}

$\mathrm{E}$

n una sociedad cada vez más orientada a disminuir el tamaño del

Estado y sus ámbitos operativos de control, la agenda educativa gubernamental está definiendo el nuevo perfil del sistema educativo formal, así como, sus objetivos sociales como formador de recurso humano. Nos evocaremos en cuáles son esos nuevos perfiles profesionales que se incentivan y las habilidades sociales que se consideran oportunas al modelo económico vigente.

Este ensayo tiene como objetivo evaluar si la automatización educativa, está entendida como una educación cada vez más tecnificada, esgrimida desde la agenda estatal y la educación pública (especialmente a nivel superior universitario), puede convertir en automáticos o irreflexivos determinados procesos sociales o afectivos. A su vez, se pretende un escrutinio recogido del contexto macroeconómico que legitima dichas posturas, así como, del debate entre una noción de formación holística, y otra, cada vez más orientada en principios mercantiles.

\section{Planes gubernamentales y políticas educativas públicas: Expectati- vas de los gobiernos recientes}

A partir de la década de los sesenta se han producido en América Latina muy importantes modificaciones políticas, económicas y sociales que han provocado, entre otras consecuencias, profundas transformaciones en los sistemas educativos de la mayoría de países de la región. Ante los substanciales y diversos cambios experimentados en las sociedades latinoamericanas, la educación formal, tanto en su figura institucional como en su actividad académica, docente y de investigación, se ve enfrentada a diversos desafíos de adecuación.

Las políticas públicas educativas no se reducen a la formación de grupos de elites intelectuales. De hecho, en América Latina sólo en muy pocas instituciones y momentos de su historia la educación superior se dedicó a la formación de elites (no directamente). A pesar de ello, en lugar de disminuir la dependencia académica y tecnológica respecto de los centros mundiales, ésta ha aumentado, dificultándose 
así la posibilidad de crear sentimientos de identidad, responsabilidad e idoneidades técnicas, científicas y humanísticas relacionadas con las desigualdades nacionales y regionales. El riesgo es que se continúe y profundice el desfasaje o falta de pertinencia de las políticas gubernamentales en dicho ámbito.

Gobiernos y políticos teóricamente esperan que la educación, y en particular las universidades, les sirvan en las finalidades para las cuales se supone están calificadas. La expectativa es que contribuyan a mejorar la posición competitiva de sus países en el mercado mundial $\mathrm{y}$ en el desarrollo económico local y regional; que lleven adelante la formación de recursos humanos, el adiestramiento de personal técnico y de servicio, la producción de conocimientos en ciencia y tecnología, investigación; así como, la actualización de conocimientos existentes (humanidades, pensamiento crítico).

Actualmente las administraciones gubernamentales proponen nuevas pautas de trabajo a las políticas educativas. Todo esto es bueno, aunque no está exento de riesgos. Quizá el riesgo mayor es que las universidades se conviertan en meros "brazos del Estado". Cada vez se hace más difícil que ellas definan sus propias agendas, restringiéndose así su tradicional autonomía intelectual. Para que una universidad posea calidad académica se requiere de una amplia colección combinada de condiciones que van de las sociomateriales (infraestructura, servicios, etc.) a las curriculares. (Ruiz, 2001, p. 32). Se necesitan estrategias creativas: el diseño de divisiones de tareas, el surgimiento de otras instituciones de educación superior que pueden asumir una parte de la demanda social, así como, que las universidades puedan cumplir con las exigencias fundamentales, tanto de creación de conocimientos (investigación y desarrollo) como de la formación de jóvenes en los campos más avanzados del conocimiento.

\section{EI nuevo perfil: La educación como bien de consumo y herramienta de mercado}

En la primera década del siglo XXI, América Latina ha vivido un cambio de concepción muy marcado en la cultura académica. Los profesores y autoridades están cada vez más dispuestos a abrazar la nueva cultura de la explotación de la investigación para el lucro comercial. Se encuentra una predisposición a orientar la educación superior hacia la 
producción inmediata de beneficios económicos a través de la investigación precompetitiva, la transferencia de tecnología, el adiestramiento y reentrenamiento de profesionales. Sin embargo, como selector de proyectos para apoyo financiero el mercado introduce nuevos sesgos, estímulos, concentraciones de poder y recursos, a veces sin que las instituciones de educación superior sean conscientes de sus implicaciones hasta que es demasiado tarde (Vessuri, 1996, p. 45). Se necesita prestar mayor atención a una planificación universitaria diferente de la tradicional, flexible, permeable a las condiciones complejas y cambiantes de este contexto.

Dichos cambios continuos en la economía, política y cultura, originados por la revolución de la nanotecnología y las nuevas tecnologías de la información, han propiciado nuevos modos de vida y nuevas perspectivas profesionales. Sobre todo, en lo referente al sistema educativo y sus alcances. Con la tecnología educativa se extiende la aplicación concreta de un nuevo modelo pedagógico en los centros educativos. Un modelo que exige un cambio de currículos, enfoques, propósitos, objetivos y contenidos temáticos. A pesar de que este enfoque es una opción, que en principio busca generar procesos formativos de mayor calidad, no se puede perder de vista las necesidades de la sociedad. Si bien es cierto, debe haber un equilibrio entre los factores educativos con las necesidades que exige el mundo contemporáneo, no debemos mantener una postura superficial donde, la necesidad de lograr mayor eficiencia económica, justifique dejar de lado una formación humana integral.

Con el restablecimiento de políticas estatales de corte liberal, el crecimiento económico, así como el aumento de valor de bienes y servicios producidos por la economía, el mercado, se ha vuelto de nuevo, un eje primordial de las políticas públicas. El crecimiento suele calcularse en términos economicistas. Pero la educación no es una mercancía cualquiera. Con la educación superior, incluso si se sabe cuánto se está pagando (...) no se sabe qué se está obteniendo con la compra (Garnier, 2006, p. 145).

Las políticas educativas de muy largo plazo han sido vistas como desventajosas a esas posturas de mercado, debido que, la capacidad productiva es en pleno empleo y por ende genera una capacidad máxima de producción. Bajo estos términos fríos, ¿cómo se debe entender la inversión social que la educación representa en sí misma? La inversión, concretamente en términos macroeconómicos consiste en posponer al futuro un posible consumo en el presente. Quien invierte y ahorra sólo cede su posibilidad de 
consumo de hoy a cambio de una adecuada compensación. Las tensiones entre lo "barato" y lo "mejor" - lo más efectivo, sustentable, estratégico, etc. - son enormes y fuente permanente de ambigüedades y contradicciones tanto en el discurso como en la realidad (Torres, 2006, p. 21).

Este proceso va dirigido una determinada visión de desarrollo, desde la búsqueda de creación de bienes para fondos de educación y tecnología, así como, el crecimiento infraestructural y mayores índices de capital. Hablamos de una educación dirigida hacia el fin de acumular factores de producción en beneficio de una industrialización pero esto, no necesariamente, rompe con la necesidad de importar conocimiento y herramientas tecnológicas.

En los países ricos, al darse un nivel per cápita alto, se genera una acumulación de capital que les permite desplegar nuevas tecnologías y que el progreso tecnológico consista en un aumento de la productividad e inversión que vuelve a regenerar dicho proceso. En estos países, se mantienen políticas de invertir en investigación y conocimiento que posteriormente se exporta a los países en vías en desarrollo, y dicha acción, hace que el capital vuelva a los mismos países vanguardistas en ciencias aplicadas. Un círculo vicioso postcolonial.

¿Por qué decimos esto? En lo que respecta a los países en vías de desarrollo, existen aún carencias en medios de transporte e infraestructura comercial que hagan ágil su economía, por lo cual, la política pública en estos países consiste en importar servicios y tecnología que lo haga competitivo en el plano económico. Este tipo de crecimiento va encaminado a una dependencia material con los países con mayor potencial económico. De esta manera, las políticas educativas recientes se direccionan, a la creación de mano obra calificada a ese proceso. Las políticas de atracción se balancean entre la llegada masiva de empresas internacionales y la casi anulación de los aranceles, haciendo que la realidad social y económica de la región se vea empañada fuertemente.

Las políticas deben impulsar mejoras significativas a las estructuras que conforman el Estado; en el plano social es necesaria la búsqueda de una política que regule la interacción entre Estado y empresa -norma que respaldaría al ciudadano-. Las directrices jurídicas deben hacer respetar el código de trabajo y las garantías sociales, apéndices que son altamente violadas en las zonas francas y por las multinacionales, con esto se hace necesario que muten los patrones políticos y legales hacia las compañías foráneas. 
Ante este contexto, es donde las políticas públicas educativas deben ser foco de conocimiento para desacelerar la importación de tecnologías con la intención de fortalecer el desarrollo interno empresarial, tecnológico y técnico. La educación como desarrollo humano se debe enfocar en dos vertientes: una, la educación como fuente de realización humana integral y emancipadora. Ésta, como medio universalizador efectivo para el acceso a la información y al desarrollo humano mediante un proceso pedagógico de calidad, que solvente la necesidad de nuevos saberes y habilidades en la población de cara al nuevo siglo. Y dos, la educación debe ser un ámbito que otorgue igualdad de oportunidades y permita compensar la marginación social y la desigualdad.

Ambas, deben encaminarse para enfocar el panorama unidireccional de la aldea global y los albores de la posmodernidad en el contexto educativo contemporáneo, así como, la diversificación de demandas productivas y necesidades sociales. Se debe interponer ante todo, el compromiso de una educación de carácter abierto y formadora de criterio, la cual permita, en los ciudadanos del siglo XXI, el desarrollo de un pensamiento analítico y crítico que estimule la instauración de un ambiente de interculturalidad y de aceptación del otro en sociedad y a las múltiples verdades.

La economía neoliberal transforma la competitividad como el máximo criterio sobre todos los valores e impone, como producto de la entrega ciega a la lógica del consumo, sus directrices de eliminación de las distorsiones del mercado (incluyendo a la educación en este ámbito). Esto lo tienen muy claro los ideólogos de la política educativa costarricense al suponer que (...) las instituciones de educación superior en América Latina deberían producir técnicos, (...) una función fuertemente ligada a las demandas del mercado laboral... (Garnier, 2006, p. 153).

A partir de esta situación, hay que pensar las alternativas, de las que aún no se tiene claridad alguna. Ahora nada más es posible afirmar que una acción disyuntiva sólo puede consistir en una acción asociativa, que por supuesto implica una acción solidaria. No más así podrían disolverse estas fuerzas compulsivas que resultan precisamente de la supresión de cualquier acción asociativa. Y hoy, tal acción asociativa no puede ser efectiva si no desemboca también en dimensiones globales. 


\section{Contexto vigente: Las políticas macroeconómicas que definen el proyecto educativo contemporáneo}

En muchos países latinoamericanos, este nuevo enfoque de los fines de la educación ha sido posible porque fue asumido como estrategia por los gobiernos. El Estado sumó a sus filas, técnicos formados en el funcionalismo de los organismos internacionales y tomaron como único consejo las políticas recomendadas por el Fondo Monetario Internacional y el Banco Mundial: Es cierto que esas politicas se dirigen en primer término a achicar los gastos del Estado para derivar los ingresos hacia el pago de la deuda externa, pero tan remanda afirmación no alcanza ni para explicar sus modelos educativos, ni, convertida en eslogan, para oponer alternativas democráticas (Puiggrós, 1996, p. 56).

Las estrategias neoliberales dirigidas a la educación imprimen a los discursos la tónica de su lógica económica. El efecto es un deterioro profundo de los enunciados dirigidos a enseñar y aprender. La educación no es una esfera desconectada del resto de la sociedad sino una modalidad de la vida social, un registro específico de los discursos sociales. Pero sus propios ritmos, tiempos, rituales y metas son triturados, cuando se los fuerza para volverlos coextensivos a los económicos o políticos.

La categoría calidad es usada por el discurso neoliberal como un instrumento de legitimación para la aplicación de premios y castigos en la tarea de disciplinar a la comunidad educativa. En ese sentido, la discusión sobre calidad académica está conceptualmente más cerca de la "eficacia" que de la "eficiencia" aunque esta última debe recibir mucha atención. Podría lograrse eficazmente los objetivos, por ejemplo, de obtener buenos profesionales, pero a través de procesos ineficientes (excesivos periodos de tiempo, derroche de recurso humano y materiales, etc.); o podría obtenerse importantes resultados de investigaciones en plazos muy largos (que otras personas o instituciones lograrian en menos tiempo) (Ruiz, 2001, p. 78).

El neoliberalismo ha roto con aquel conjunto de tradiciones, pero debe usar algunos de sus enunciados para adquirir legitimidad. Sus reformas avanzan cobijadas en promesas tales como mejorar la inversión educativa y hacer más eficiente el gasto con el fin de lograr una equidad en la provisión de educación a la población. 


\section{Alternativas: La socialización, no la automatización}

El paradigma constructivista humanista e integral, y de un enfoque eminentemente cualitativo, presenta entre sus principales percepciones filosóficas, que el ser humano está en un continuo proceso de desarrollo, donde reducir el comportamiento del individuo no es aceptable. Los sujetos deben de desenvolverse integralmente como individuos. El ser humano, en esta perspectiva, no es la especie humana o una noción general, sino el individuo humano considerado en su absoluta singularidad.

Desde el punto de vista del neoliberal, se ha utilizado la percepción de que el individuo crea su propia personalidad por medio de las elecciones o decisiones que toma cotidianamente, ya que el humano es un ser libre. Desde el punto de vista de la fenomenología, se dice que es el entendimiento o estudio de la percepción externa o interna como un acontecimiento subjetivo, sin ningún tipo de a priori, donde el individuo se rige a través de su propia percepción subjetiva, donde responde al ambiente a como uno lo percibe y comprende, donde es necesario entender al otro en sus procesos psicológicos, es necesario comprender la problemática desde su punto de vista y no desde otra perspectiva.

Los alumnos deben tener una iniciativa que lo desarrolle con autodeterminación e individualidad; el papel del maestro debe darse con sus alumnos en una relación basada en el respeto con sus ideales propios, el cual debe de potencializar y fomentar las necesidades de sus estudiantes. Un clima social fundamental para que la comunicación de la información académica y emocional sea exitosa, es que la metodología de enseñanza, debe ser abierta a nuevas experiencias, donde se fomente el cooperativismo entre los sujetos.

La necesidad imperativa de revertir el modelo liberal de educación por una educación integral para lograr el desarrollo total de la persona, involucra una revisión del currículo y el uso de ejes transversales que respondan a las necesidades del contexto. Según la teoría, el proceso de aprender necesita recursos de tipo tecnológico, en este caso dentro de:

1. La concepción educativa que fundamenta el acceso de esos recursos en el espacio educativo.

2. La disposición que brinde el docente en utilizar estos recursos y la facilidad de acceso a ellos. 
De esta manera, se habla de interacción con los recursos tecnológicos, indicando la complejidad de la construcción del proceso de enseñanza -aprendizaje y la problemática de socialización de los seres humanos que esto implica. Lo anterior porque, el aprendizaje depende del desarrollo biológico y el desarrollo de las operaciones cognoscitivas que se apoyan en la interrelación activa del individuo con el entorno; es decir, el desarrollo cognoscitivo se sustenta en el proceso de adquisición de un andamiaje que la máquina por sí sola no ofrece. Las características de los enfoques mencionados establecen que la interacción social se da por medio de situaciones donde se da la relación, la comunicación del individuo, por tanto, los medios tecnológicos deben ser utilizados no en demasía.

Si se debe admitir que en un espacio educativo con recursos tecnológicos hay un dinamismo constante que genera un intercambio entre el alumno y los recursos. Para seguir dentro de la misma temática; un espacio educativo con recursos tecnológicos ofrece una conflicto de contextos que se interrelacionan entre sí, alrededor de las experiencias y significaciones construidas. Al igual que lo anterior, se da la interacción de otros contextos como: el sociocultural, el intersubjetivo, afectivo cognitivo, entre otros.

Los supuestos pedagógicos de un ambiente de aprendizaje con recursos informáticos dependen de la visión pedagógica que se quiere ofrecer a determinado ambiente, se plantea que los recursos tecnológicos poseen una relación estrecha con el ser humano, es decir, provienen de un contexto donde se da la relación humano- máquina, por ello estos supuestos planean que no se pueden dejar de lado el aprender por medio de estos nuevos recursos.

La persona se construye mediante las interacciones y la interlocución con otras personas y con los objetos: en síntesis cuando una persona intercambia pensamientos con otras, tiene la necesidad de un marco de referencia común a partir del cual es posible la comprensión mutua. La cultura se aprecia como mediadora entre las potencialidades de las personas y su realidad en la creación de un proceso continuo de comunicación que les permiten dialogar el contexto. En este espacio de intercambios, los sujetos intervienen sobre sí mismos y sobre la sociedad porque elaboran sus propias percepciones por medio del lenguaje, que es la herramienta más importante para la construcción del aprendizaje. En ese sentido, las computadoras pueden manejarse como 
herramientas de aprendizaje, porque proveen recursos que pueden utilizarse apropiadamente y en función de metas, intereses y posibilidades que tenga el sujeto.

Por ello el aprendizaje virtual es un intento de implementar los procesos de aprendizaje y enseñanza mediante aplicaciones telemáticas que garanticen la calidad de la comunicación en la formación presencial o a distancia. Pero que en la realidad macroeconómica del neoliberalismo de los últimos treinta años, se sustituye todo este proceso por una automatización gradual de los procesos cognitivos, minando así, las relaciones sociales que supone en sí misma la educación.

\section{Conclusiones}

En este mundo interconectado, se está haciendo evidente una creciente valorización del conocimiento, así como la convicción de que la capacidad de las personas para crear, apropiarse y adaptar nuevas tecnologías, se ha convertido en elemento estratégico para incrementar la competitividad. Algunos gobiernos latinoamericanos comienzan a percibir la educación como el motor principal del desarrollo. Las relaciones entre los dos actores principales de la educación latinoamericana en la primera mitad del siglo XX, es decir, las universidades y los Estados, han variado de manera sustancial; se necesita establecer hoy un nuevo diálogo, tanto entre ellos como con otros importantes actores sociales, para lograr una estrategia consensuada que pueda hacer realidad las transformaciones necesarias.

Queda claro que la transformación de las políticas públicas educativas debe ser realizada en su propio seno, con un fuerte compromiso y participación de las comunidades que las sostienen, en consonancia, con los cambios de las sociedades que les rodean, buscando la preservación de su identidad y su cultura.

Es indispensable que se vigilen las reformas neoliberales para resguardar los aspectos que aún están en pie de los sistemas educativos latinoamericanos. Al mismo tiempo, es necesario diseñar políticas de cambio pedagógico que respeten tres características: participación, experimentación y gradualidad. Las reformas educacionales son procesos profundos, que ponen en movimiento aspectos estructurales de la vida social y cultural, que comprometen el imaginario colectivo, la memoria histórica y la prospectiva. 
No son posibles sin consenso de la comunidad educativa y es necesario analizar las experiencias que la sociedad fue acumulando y aprender de ellas. Las políticas impuestas verticalmente en forma homogénea e inconsulta a numerosos países constituyen probables fracasos y requieren de medidas autoritarias para sostenerse.

Las innovaciones introducidas deben experimentarse, los modelos pedagógicos deben sufrir la hibridación a la cual lo somete su confrontación con los procesos educacionales específicos. Una reforma responsable debe ser respetuosa de los tiempos y modalidades culturales, de las demandas específicas y del derecho de los sujetos de la comunidad educativa a participar de la reforma de su educación, la de sus hijos o la que los compromete como docentes.

\section{Referencias bibliográficas}

Garnier, L. (2006). Conocimiento y educación superior en América Latina: ¿Mercancías incómodas? En V. Muñoz. (comp.), El oro por las cuentas: Miradas a la mercatilización de la educación. Heredia: Centro de Investigación y Docencia en Educación. Editorial Luna Híbirida.

Puiggrós, A. (1996). Educación neoliberal y quiebre educativo. Revista Nueva Sociedad,146, 90-101. Recuperado de http://www.nuso.org/upload/articulos/2549 1.pdf

Ruiz, A. (2001). La Educación Superior en Costa Rica. San José: EUCR.

Torres, R. M. (2006). ¿Mejorar la calidad de la educación básica? Las estrategias del banco mundial. En V. Muñoz. (comp.), El oro por las cuentas: Miradas a la mercatilización de la educación. Heredia: Centro de Investigación y Docencia en Educación. Editorial Luna Híbirida.

Vessuri, H. (1996). Pertinencia de la educación superior latinoamericana a finales del siglo XX. Revista Nueva Sociedad, 146, 102-107. Recuperado de http://www. nuso.org/upload/articulos/2550_1.pdf 